\title{
Gastric Cancer by AJCC v8 Stage
}

National Cancer Institute

\section{Source}

National Cancer Institute. Gastric Cancer by A/CC v8 Stage. NCI Thesaurus. Code C133638.

A term that refers to the staging of gastric cancer according to the American Joint Committee on Cancer, 8th edition. 